\title{
FENOLOGIA DE PAEPALANTHUS HILAIREI KOERN., $P$. POLYANTHUS (BONG.) KUNTH E P. ROBUSTUS SILVEIRA: PAEPALANTHUS SECT. ACTINOCEPHALUS KOERN. - ERIOCAULACEAE ${ }^{1}$
}

\author{
Paulo Takeo Sano ${ }^{2}$
}

Recebido em 31/10/96. Aceito em 24/12/96.

\begin{abstract}
RESUMO - (Fenologia de Paepalanthus hilairei Koern., P. polyanthus (Bong.) Kunth e $P$. robustus Silveira: Paepalanthus sect. Actinocephalus Koern. - Eriocaulaceae). Foi realizado o acompanhamento fenológico de três espécies de Paepalanthus sect. Actinocephalus: $P$. hilairei, $P$. polyanthus e $P$. robustus. Realizaram-se viagens mensais à Serra do Cipó, Minas Gerais, Brasil, durante um período de 21 meses. Detectou-se que $P$. hilairei e $P$. robustus são perenes e $P$. polyanthus é bienal. Nestas espécies verificou-se um período de floração concentrado nos meses do verão. A ocorrência de odor é registrada pela primeira vez em representantes do gênero.
\end{abstract}

Palavras-chave: Eriocaulaceae, Paepalanthus, Actinocephalus, fenologia, Serra do Cipó

\begin{abstract}
Phenology of Paepalanthus hilairei Koern., $P$. polyanthus (Bong.) Kunth and $P$. robustus Silveira: Paepalanthus sect. Actinocephalus Koern. - Eriocaulaceae). Phenological studies of three Paepalanthus sect. Actinocephalus species were done - P. hilairei, $P$. polyanthus and $P$. robustus. Monthly expeditions for 21 months to Serra do Cipó, Minas Gerais, Brazil were realized. It was detected that $P$. hilairei and $P$. robustus are perennial plants and $P$. polyanthus is a biennial plant. All of them present the blooming period mainly in Summer. Smell occurrence is registred in Paepalanthus for the first time.
\end{abstract}

Key-words: Eriocaulaceae, Paepalanthus, Actinocephalus, phenology, Serra do Cipó

\section{Introdução}

Predominantemente herbáceas e com distribuição pantropical, as Eriocaulaceae incluem cerca de 1200 espécies reunidas em dez gêneros (Hensold \& Giulietti 1991). Segundo Giulietti (1978) é uma família bem caracterizada pelas inflorescências

\footnotetext{
Parte da dissertação de Mestrado realizada no Depart. de Botânica - USP, sob orientação da Profa. Dra. Ana Maria Giulietti. Financiamento do $\mathrm{CNPq}$

2 Depart. de Botânica, Instituto de Biociências, Universidade de São Paulo. C.P. 11461, CEP 05422-970

São Paulo, SP, Brasil
} 
capituliformes. Paepalanthus Kunth é o maior gênero, com 485 espécies, distribuindo-se quase exclusivamente nas Américas Central e do Sul. Há cinco espécies endêmicas da África e duas espécies disjuntas entre esse continente e as Américas (Giulietti \& Hensold 1990). Para o Brasil são referidas 407 espécies, das quais 67\% encontram-se restritas a Minas Gerais. Entre os taxa infra-genéricos Paepalanthus sect. Actinocephalus representa um grupo muito bem delimitado e bastante característico pelo arranjo umbeliforme dos escapos e pela presença de estigmas simples, quando a quase totalidade das espécies do gênero apresenta estigmas bífidos. Sano (1993) comenta a necessidade de uma reavaliação completa desse grupo e de sua posição taxonômica dentro do gênero.

Trabalhos recentes sobre Paepalanthus têm sido realizados sob uma abordagem taxonômica (Hensold 1988, Sano 1993), anatômica (Castro 1986), fitoquímica (Vilegas et al. 1990) e biogeográfica (Giulietti \& Meikle 1982). Entretanto, não apenas no gênero, mas também na família, há grande carência de estudos voltados para a biologia e reprodução das espécies. Isso ocorre, em parte, porque ainda no aspecto taxonômico há muitas questões a serem resolvidas, de maneira a permitir estudos mais detalhados e abrangentes.

O presente trabalho tem por objetivo apresentar e discutir os dados obtidos sobre a fenologia e o ciclo de vida de três espécies de Paepalanthus sect. Actinocephalus. Com isso espera-se ampliar o número de informações sobre os campos rupestres brasileiros e abrir caminhos para novas pesquisas sobre este tema.

\section{Material e métodos}

Foram analisadas três espécies de Paepalanthus sect. Actinocephalus: Paepalanthus hilairei Koern., P. polyanthus (Bong.) Kunth e $P$. robustus Silveira, ocorrentes na Serra do Cipó, município de Santana do Riacho, Minas Gerais (19¹0' - 19²0'S e $\left.43^{\circ} 30^{\prime}-43^{\circ} 40^{\prime} \mathrm{W}\right)$. Justifica-se a escolha dessas espécies por ocorrerem em agrupamentos relativamente abundantes em toda a extensão da Serra.

Os dados foram coletados em viagens mensais à Serra do Cipó, de julho de 1991 a março de 1993, com permanência média de quatro dias por mês. Foi realizada uma viagem prévia de observação e reconhecimento, em março de 1991 e outra posterior, para comprovação de alguns dados, em novembro de 1993.

Foram acompanhadas duas subpopulações de Paepalanthus hilairei $(\mathrm{Ph} 1 \mathrm{e}$ $\mathrm{Ph} 2)$, duas de $P$.polyanthus ( $\mathrm{Pp} 1 \mathrm{e} \mathrm{Pp} 2)$ e uma de $P$. robustus, em áreas adjacentes à rodovia MG 010, que liga Belo Horizonte a Conceição do Mato Dentro, tendo sido marcados 10 indivíduos de cada subpopulação.

Os indivíduos foram marcados com etiquetas metálicas numeradas. Para as medições foi utilizada fita métrica com escala em centímetros. Os dados foram anotados em tabelas de acordo com as seguintes padronizações: nas medidas de diâmetro da roseta, consideraram-se apenas as folhas verdes, tomando-se dois eixos ortogonais de medidas, de um extremo a outro da roseta e anotando-se a média das medições. Para a altura considerou-se, nas plantas em roseta, o comprimento desde o 
solo até o ápice do caule. No caso de Paepalanthus polyanthus, quando em estádio reprodutivo, considerou-se o comprimento desde o solo até o ápice do eixo da sinflorescência. Mediu-se também o comprimento médio dos paracládios.

Além destes parâmetros, comuns a todas as espécies, foram observados outros exclusivos para cada uma:

Em Paepalanthus hilairei, além da altura, mediu-se o crescimento do ápice após os ciclos de florada, o número de paracládios e o comprimento médio dos paracládios. Em $P$. polyanthus foi contado o número de paracládios secos portando inflorescências velhas e medida a altura das plantas secas. Foram selecionadas as que apresentavam plantas jovens na base, cujo crescimento foi acompanhado. Foi ainda observada a porcentagem de folhas verdes e secas. Em $P$. robustus mediu-se o número de paracládios por planta.

Foram elaborados tabelas e gráficos de crescimento vegetativo e de fenologia. Para Paepalanthus polyanthus foi elaborado um gráfico de porcentagem média de folhas secas e para $P$. robustus foi feito um gráfico da porcentagem de indivíduos reprodutivos. As fases fenológicas de cada espécie, mensalmente, ao longo de um ano são apresentadas em gráfico. Foram definidas cinco fases fenológicas para os indivíduos:

1. vegetativa: quando estavam ausentes quaisquer elementos reprodutivos.

2. paracládios em desenvolvimento: quando os paracládios se tornavam visíveis na axila das folhas até o início do desenvolvimento dos escapos.

3. capítulos fechados: desde o desenvolvimento dos escapos, que portavam no ápice capítulos jovens, até o início da abertura das brácteas involucrais.

4. antese: a partir da abertura das brácteas involucrais e das flores mais periféricas, quando podiam ser vistas flores expondo anteras.

5. dispersão: quando mais de $50 \%$ das flores pistiladas de um capítulo dispersavam seus frutos.

Para padronização dos termos utilizados para os arranjos das inflorescências, seguiram-se os conceitos propostos por Weberling (1989) para as inflorescências compostas. As demais estruturas reprodutivas e vegetativas seguem as denominações utilizadas por Giulietti (1978, 1984), Hensold (1988) e Giulietti \& Hensold (1990). O termo "fenologia" foi considerado com a definição dada por Lieth (1974).

\section{Resultados}

\section{Paepalanthus hilairei}

Em Paepalanthus hilairei nas duas subpopulações as plantas apresentaram crescimento de cerca de $5,0 \mathrm{~cm}$ ao mês, sendo que a partir de novembro, 80 a $90 \%$ dos indivíduos haviam iniciado o desenvolvimento de novos paracládios. Durante a fase reprodutiva, desenvolveram, em média, cinco paracládios por indivíduo e concomitante a isso, o ápice caulinar seguiu seu crescimento.

A figura 1 ilustra a fenologia de Paepalanthus hilairei. Nos meses de dezembro e janeiro os paracládios atingiram o comprimento médio de $12,5 \mathrm{~cm}$ e passaram a 
desenvolver escapos, cujo crescimento ocorreu de um mês a outro. Em janeiro, os capítulos iniciaram a abertura das brácteas involucrais e, em fevereiro, todos apresentavam flores em antese, exalando perfume suave e adocicado nas primeiras horas da tarde. As flores estaminadas eram facilmente perceptíveis nos capítulos por apresentarem o tubo da corola bem distendido e as anteras expostas. Em março, todos os indivíduos se encontravam no início da fase de dispersão (Fig. 1). A maturação das flores em um mesmo capítulo ocorre centripetamente. Inicialmente abrem as flores periféricas, gradativamente amadurecem as intermediárias e finalmente as mais centrais.

Abr Mai Jun Jul Ago Set Out Nov Dez Jan Fev Mar

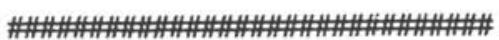
IIIIIIIIIIIIIIIIIIIIIIIIIIIIIIIIII $\gg \gg \gg \gg>\gg>>>$ 0000000000000000000000000 0000000000000 (n)

Figura 1. Fenologia de 20 indivíduos de Paepalanthus hilairei no período de abril de 1992 a março de 1993. Subpopulações do km $108(\mathrm{Ph} 1)$ e km $124(\mathrm{Ph} 2)$ da rodovia MG 010 (Belo Horizonte - Conceição do Mato Dentro), Serra do Cipó, MG, Brasil. \#\#\# vegetativo; /l/ paracládios em desenvolvimento; >> capítulos fechados; ooo capítulos em antese; *** capítulos em dispersão.

Paepalanthus hilairei se apresenta com ciclo de vida perene, com crescimento contínuo do ápice caulinar. Como o crescimento dos paracládios ocorre uma vez ao ano, no início do verão, pode-se inferir aproximadamente portanto, a idade dos indivíduos. Foi encontrado um indivíduo com paracládios secos ou quebrados em até cinco posições diferentes do caule, indicando cinco períodos reprodutivos em anos anteriores.

Um evento bastante freqüente foi a podridão e morte do ápice caulinar. Com a destruição do ápice, desenvolveram-se a partir da região subapical diversos ramos laterais com numerosas raízes adventícias em sua base. Estes ramos originaram posteriormente novos paracládios e inflorescências. Durante o período de observação não foi verificado o desprendimento desses ramos da planta-mãe, porém observou-se que, com seu desenvolvimento, há aumento de peso na porção apical da planta, curvando-a.

Em ambas as subpopulações verificou-se um período longo de floração e dispersão (Fig. 1). 


\section{Paepalanthus robustus}

Os indivíduos de Paepalanthus robustus não apresentaram crescimento significativo ao longo do ano, caracterizando um crescimento lento quando comparado ao das demais espécies.

A porcentagem de indivíduos vegetativos de $50 \%$ no final do período das chuvas, passou a 70\% durante o inverno e o estio (Fig. 2). Em outubro, após as primeiras chuvas, $90 \%$ dos indivíduos apresentavam paracládios jovens com número variável entre cinco e 24 por indivíduo e comprimento médio de $16,0 \mathrm{~cm}$. Em dezembro, $80 \%$ dos indivíduos encontravam-se em flor. Em janeiro, esta porcentagem foi encontrada para indivíduos em fase de dispersão, muito embora essa fase tenha sido verificada em maior ou menor grau durante o ano todo (Fig. 3). Embora a subpopulação tenha estado em flor durante o ano todo, observou-se que a floração mais intensa coincidiu com o início da estação chuvosa.

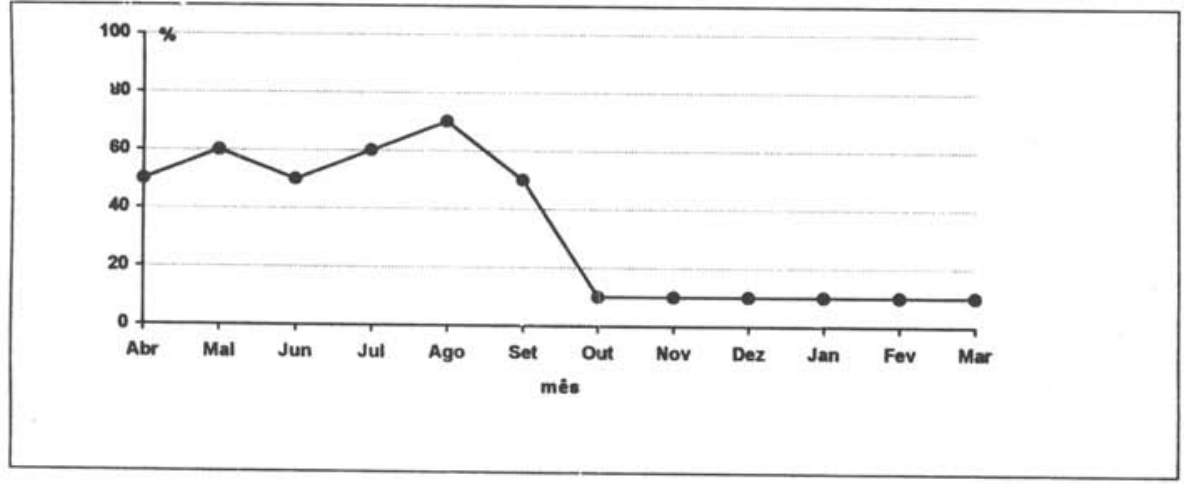

Figura 2. Porcentagem de indivíduos vegetativos de Paepalanthus robustus no período de abril de 1992 a março de 1993. Subpopulação do km 125 da rodovia MG 010 (Belo Horizonte - Conceição do Mato Dentro), Serra do Cipó, MG, Brasil.

Paepalanthus robustus apresenta ciclo de vida perene, pois os indivíduos tiveram ciclos vegetativos e reprodutivos seqüentes, sem que houvesse morte. Durante todo $\mathrm{o}$ ano foram verificados indivíduos em todas as fases fenológicas, desde plantas vegetativas até em dispersão.

\section{Paepalanthus polyanthus}

Paepalanthus polyanthus apresenta ciclo de vida bienal, característica pouco comum em plantas tropicais. Em observações realizadas desde março de 1991, constatou-se que subpopulações cujos indivíduos se encontravam muito jovens, não floresceram naquele ano, apresentando-se reprodutivos somente no final de 1992, secando posteriormente. Durante os primeiros doze meses, as plantas apresentaram 
Abr Mai Jun Jul Ago Set Out Nov Dez Jan Fev Mar

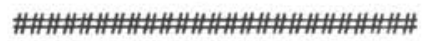

IIIIIIIIIIIII

- IIIIIIIIIIIIIIIIIIIIIIIIIIIIIII

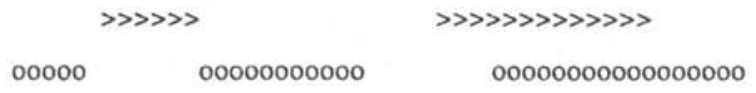

Figura 3. Fenologia de 10 indivíduos de Paepalanthus robustus no período de abril de 1992 a março de 1993. Subpopulação km 124 da rodovia MG 010 (Belo Horizonte - Conceição do Mato Dentro), Serra do Cipó, MG, Brasil. \#\#\# vegetativo; I// paracládios em desenvolvimento; 》 capítulos fechados; ooo capítulos em antese; *** capítulos em dispersão.

exclusivamente crescimento vegetativo. A partir do segundo ano (setembro), ocorreu um grande aumento na produção de folhas da roseta e, coincidindo com o início da estação chuvosa, houve rápido desenvolvimento de um eixo central portando os paracládios com numerosos escapos. A seguir houve a formação e antese das flores entre novembro e dezembro - sendo que a formação de frutos e a dispersão ocorreu em janeiro (Fig. 4), coincidindo com maior volume de chuvas, o que pode estar associado à presença de condições propícias à germinação. Simultaneamente a estes eventos, as folhas foram se tornando marcescentes e os indivíduos, ao final da dispersão, encontravam-se secos.

Os indivíduos de uma das subpopulações (Pp1) apresentaram, ao longo do ano, grande variação no diâmetro da roseta, devido a uma forte queimada durante o mês de agosto de 1992 (Fig. 5). Todas as folhas mais externas foram queimadas, permanecendo verdes somente as mais centrais, que se desenvolveram rapidamente após o início das primeiras chuvas, em setembro. A partir de outubro, houve grande desenvolvimento do caule e reconstituição do diâmetro original da roseta (Fig. 5). Estes eventos precederam o desenvolvimento dos paracládios, em média de 31 por planta, com comprimento médio de $7,5 \mathrm{~cm}$. Simultaneamente à maturação dos frutos e sua dispersão, as folhas dos indivíduos de Paepalanthus polyanthus tornaram-se amarelas e senescentes e em março encontravam-se secas (Fig. 6), ocasião em que todos os indivíduos apresentavam frutos em dispersão.

Nesta espécie, ao contrário de Paepalanthus hilairei, não ocorre continuidade do crescimento vegetativo após a floração. O ápice caulinar se alonga num eixo e dá origem aos paracládios, apresentando logo a seguir, sinais de ressecamento e morte. Isso ocor- 
Abr Mai Jun Jul Ago Set Out Nov Dez Jan Fev Mar

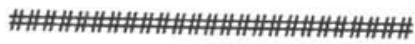

IIIIIIIIIIIII

$\gg>>>$

00000000000

(1)

Figura 4. Fenologia de 10 indivíduos de Paepalanthus polyanthus no período de abril de 1992 a março de 1993. Subpopulação do km 125 (Ppl) da rodovia MG 010 (Belo Horizonte - Conceição do Mato Dentro), Serra do Cipó, MG, Brasil. \#\#\# vegetativo; /// paracládios em desenvolvimento; $\gg>$ capítulos fechados; ooo capítulos em antese; ${ }^{* * *}$ capítulos em dispersão.

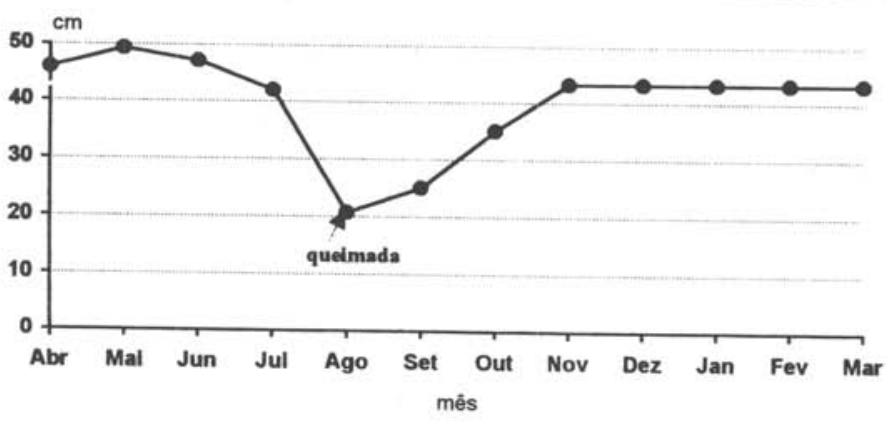

Figura 5. Médias do diâmetro da roseta de 10 indivíduos de Paepalanthus polyanthus no período de abril de 1992 a março de 1993. Subpopulação do km 125 (Ppl) da rodovia MG 010 (Belo Horizonte - Conceição do Mato Dentro), Serra do Cipó, MG, Brasil.

reu não apenas nos indivíduos marcados, como também nos demais observados ao acaso, com exceção de um, no qual o ápice terminou em uma nova coflorescência.

Na subpopulação Pp2 os indivíduos foram marcados no estádio em que se encontravam secos e havia plantas surgindo na base das rosetas. Tendo sido revolvido o solo ao redor dos indivíduos secos, observaram-se plântulas em diferentes estádios, não ocorrendo, porém, ligação entre os indivíduos jovens e a planta seca. Esses mesmos indivíduos jovens, que em 1992 se encontravam vegetativos, foram encontra- 


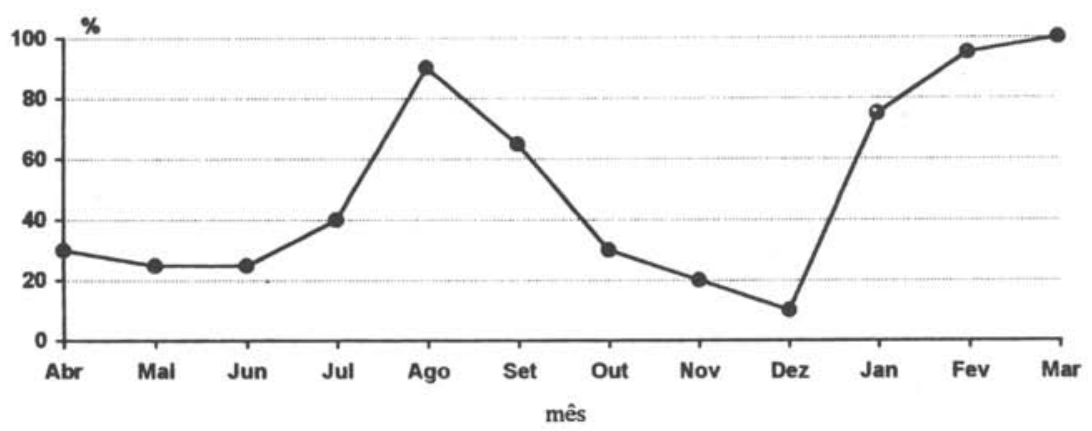

Figura 6. Porcentagem de folhas secas em 10 indivíduos de Paepalanthus polyanthus no periodo de abril de 1992 a março de 1993. Subpopulação do km 125 (Pp1) da rodovia MG 010 (Belo Horizonte - Conceição do Mato Dentro), Serra do Cipó, MG, Brasil.

dos em novembro de 1993 e todos se apresentavam reprodutivos, fenecendo logo em seguida.

Nos capítulos de Paepalanthus hilairei, $P$. polyanthus e $P$. robustus verificouse a presença de odor suave e adocicado no final da manhã e nas primeiras horas da tarde. Até por volta de 10:00h não era possível detectar odor. A partir desse horário, o odor come-çava a ser perceptível e se intensificava, alcançando o máximo de intensidade entre 12:00 e 14:00h, podendo ser sentido, ainda que tenuemente, até as 17:00h. Associa-se a esse odor a observação de alguns Coleoptera, Diptera e Hymenoptera nas flores das espécies referidas. Como a permanência desses insetos nas flores era muito rápida $(1-2 \mathrm{~s})$ não foi possível observar o seu comportamento, de maneira a inferir algo sobre sua efetividade como polinizadores.

\section{Discussão}

Em Paepalanthus hilairei, o desenvolvimento dos paracládios foi relativamente rápido, sendo bastante característica a disposição verticilada destes paracládios no caule. Isso marca ciclos de floração bem definidos e confere à planta um porte exclusivo que permite a distinção dessa espécie em campo, mesmo à distância. $\mathrm{O}$ período em que há flores em antese foi o mais longo (oito meses) verificado entre as espécies estudadas. Anteriormente períodos de floração de seis meses haviam sido os mais longos entre os observados por Faria (1994) para espécies de diversas outras famílias na Serra do Cipó.

Em ambas as subpopulações, verificou-se um longo período de floração e dispersão, possivelmente decorrentes da maturação centrípeta dos capítulos. A dispersão por um espaço maior de tempo possibilita a germinação de sementes e o surgimento de novos indivíduos por um período de tempo mais longo. 
Quanto ao desenvolvimento de gemas laterais após a destruição do ápice caulinar, embora não tenha sido verificado o desprendimento desses ramos com raízes adventícias, foi observado o curvamento da planta-mãe pelo peso dos ramos. Possivelmente alguns desses ramos podem passar a ter vida independente, caso toquem o solo ou a planta-mãe se quebre, representando uma forma alternativa de reprodução vegetativa.

A presença de todas as fases fenológicas durante o ano todo na população de Paepalanthus robustus, representa possivelmente uma estratégia de reprodução. Souza (1990) refere o mesmo mecanismo nas fases fenológicas das espécies de Physocalyx (Scrophulariaceae) da Serra do Cipó, ocorrendo, em um mesmo mês, indivíduos com flores e botões, com frutos maduros e imaturos ou mesmo plantas vegetativas.

O fogo pode ser um fator regulador da floração, pois quando comparada com indivíduos não atingidos por queimadas em outras áreas da Serra do Cipó, a subpopulação teve um aumento significativo no número de paracládios em desenvolvimento após a ocorrência de uma queimada.

A presença de folhas secas reflexas e persistentes no caule dos indivíduos de Paepalanthus robustus podem indicar uma evidência da não passagem de fogo pela área. Mesmo nos indivíduos mais altos, pode-se verificar a ocorrência de folhas secas em toda a extensão do caule. Em áreas expostas ao fogo, as folhas são queimadas permanecendo apenas restos de bainhas e a roseta de folhas verdes na porção apical. A persistência dessas bainhas ou das folhas secas em P. robustus provavelmente representa uma adaptação de proteção contra os efeitos do fogo.

Paepalanthus polyanthus foi a espécie que apresentou as fases fenológicas melhor delimitadas. O caráter biênico encontrado nos indivíduos desta espécie parece não ser constante nas populações ao longo de sua distribuição. Segundo Castellani (1990), nas dunas da Ilha de Santa Catarina, os indivíduos de $P$. polyanthus encontrados apresentam-se monocárpicos perenes. Não obstante, o que tem sido observado em todas as populações da Serra do Cipó é um marcado intervalo de dois anos entre o início do desenvolvimento, reprodução e morte dos indivíduos. O caráter bienal pode estar relacionado ao fato de que o ambiente na Serra do Cipó talvez apresente condições que favoreçam uma periodicidade mais nítida nos eventos reprodutivos da espécie. Nos indivíduos dessa área, a maior porcentagem de folhas verdes coincide com o período de crescimento reprodutivo da planta, fato que pode ser explicado pela maior necessidade de alocação de recursos e maior esforço fotossintético para um rápido desenvolvimento do eixo, paracládios, escapos e capítulos. Castellani (1990) sugere a ocorrência de bienalidade facultativa para espécies monocárpicas perenes, estando a longevidade dos indivíduos vinculada a um tamanho mínimo para reprodução. Este valor em $P$. polyanthus seria de $15-18 \mathrm{~cm}$ de diâmetro da roseta (Castellani 1990). Tenório (1969) observou nas Gramineae de Pernambuco que o período reprodutivo era pre-cedido por aumento no desenvolvimento vegetativo e Mantovani \& Martins (1988) referem evento semelhante para todas as espécies herbáceosubarbustivas do cerrado de Moji-Guaçu (SP).

Com relação ao grande agrupamento de indivíduos jovens próximos à planta- 
mãe, o que ocorre é a germinação de sementes que caem muito próximas, originando assim, novos indivíduos. Esse evento provavelmente encontra-se associado ao mecanismo de dispersão da espécie apresentado por Sano (1993). Moldenke \& Smith (1976) e Castellani et al. (1996) também mencionam que indivíduos dessa espécie possuem distribuição agrupada.

A perda, ou o não desenvolvimento da inflorescência terminal em Paepalanthus polyanthus, caracteriza o fenômeno, denominado por Weberling (1989), de "truncamento" e considerado como uma derivação originada a partir do desenvolvimento completo da sinflorescência. Outra hipótese seria a ocorrência de danos tais como os verificados por Castellani (1990) nas inflorescências laterais, causados, entre outros fatores, pelo ressecamento e mal desenvolvimento devido à ação do vento, a interferências resultantes da sobreposição de capítulos nas umbelas e à predação de flores por lagartas (Lepidoptera, Geometridae).

$\mathrm{O}$ odor verificado nas flores das três espécies de Paepalanthus, associado à observação de insetos sobre as inflorescências, pode indicar a entomofilia como uma das possíveis síndromes de polinização. Entomofilia foi sugerida por Ruhland (1903), Hare (1950) e Cronquist $(1981,1988)$ como uma das síndromes de polinização para todas as Eriocaulaceae. Faria (1994) refere entomofilia para várias outras espécies de Paepalanthus da Serra do Cipó: P. speciosus, P. bromelioides e P. geniculatus e Hensold (1988) a refere para os indivíduos de Paepalanthus subgen. Xeractis.

De uma maneira geral, observou-se que, nas três espécies, todos os capítulos de uma mesma coflorescência apresentam simultaneidade nas fases fenológicas. Assim, não há numa coflorescência, capítulos fechados e outros já em antese ou frutificação. Essa simultaneidade provavelmente está relacionada à maior floração na estação chuvosa onde, além da maior disponibilidade hídrica para a fase reprodutiva, as águas de precipitação certamente auxiliam na dispersão dos diásporos. Posteriormente, a presença de solos úmidos é um fator que favorece a germinação das sementes e fixação das plântulas.

O arranjo umbeliforme dos escapos, característico em Paepalanthus sect. Actinocephalus, associado aos exclusivos mecanismos de dispersão dos diásporos (Sano 1993), provavelmente contribuíram para o sucesso evolutivo do grupo, facilitando a exploração, por parte de algumas espécies, de ambientes tão distintos como os campos rupestres e alpinos das serras brasileiras, as dunas e restingas litorâneas e os cerrados do Brasil Central.

A constatação do ciclo da vida perene em $P$. hilairei e Paepalanthus robustus e da bienalidade em $P$. polyanthus, além de representar dados inéditos no conhecimento da biologia de Eriocaulaceae, têm ainda implicações relacionadas à exploração comercial destas espécies. A importância econômica da família encontra-se relacionada ao seu valor ornamental, sendo comercializadas com o nome popular de "semprevivas" (Giulietti et al. 1988, De Lazzari 1995). Estes mesmos autores citam cifras da ordem de toneladas de escapos que são exportados anualmente para vários países. Pio Corrêa (1984) refere $P$. polyanthus — "sempre-viva-da-serra" — entre as espécies de Paepalanthus com importância econômica relacionada à exportação. Em Diamantina 
e Serra do Cabral (MG), P. hilairei tem sido coletada e vendida sob os nomes de "bemcasado" e "agarradinho". P. robustus, embora não tenha sido referida até o momento como explorada economicamente, apresenta sua importância ligada ao fato de ser endêmica da Serra do Cipó, não tendo sido coletada em qualquer outra região do país. Os estudos de fenologia e ciclo de vida certamente representam uma contribuição significativa para quaisquer tentativas de manejo ou cultivo destas espécies.

\section{Referências bibliográficas}

Castellani, T.T. 1990. Aspectos da ecologia reprodutiva de Paepalanthus polyanthus (Bong.) Kunth nas dunas da Joaquina, Ilha de Santa Catarina, SC. Anais do II Simpósio de Ecossistemas da Costa Sul e Sudeste Brasileira: estrutura, função e manejo. Águas de Lindóia. ACIESP 3:488-498.

Castellani, T.T.; Vieira, S. \& Scherer, K.Z. 1996. Contribuição ao conhecimento da distribuição espacial de Paepalanthus polyanthus (Bong.) Kunth (Eriocaulaceae) em áreas de baixada úmida de dunas. Acta bot. bras. 10(1):25-36.

Castro, N.M. 1986. Estudos morfológicos dos órgãos vegetativos de espécies de Paepalanthus Kunth (Eriocaulaceae) da Serra do Cipó (Minas Gerais). Dissertação de Mestrado. São Paulo: Universidade de São Paulo.

Cronquist, A. 1981. An integrated system of classification of flowering plants. Columbia University Press. New York.

Cronquist, A. 1988. The evolution and classification of flowering plants. 2nd. ed. The New York Botanical Garden. New York.

De Lazzari, L.R.P. 1995. Syngonanthus Ruhland (Eriocaulaceae) na Serra do Cipó, Minas Gerais, Brasil. Dissertação de Mestrado. São Paulo: Universidade de São Paulo.

Faria, G.M. 1994. A flora e a fauna apícola de um ecossistema de campo rupestre, Serra do Cipó, MG, Brasil: composiçāo, fenologia e suas interaçōes. Tese de Doutorado. Rio Claro: Universidade Estadual Paulista (UNESP).

Giulietti, A.M. 1978. Os gêneros Eriocaulon L. e Leiothrix Ruhl. na Serra do Cipó, Minas Gerais, Brasil. Tese de Doutorado. São Paulo: Universidade de São Paulo.

Giulietti, A.M. 1984. Estudos taxonômicos no gênero Leiothrix Ruhl. (Eriocaulaceae). Tese de LivreDocência. São Paulo: Universidade de São Paulo.

Giulietti, A.M. \& Hensold, N.C. 1990. Padrões de distribuição geográfica dos gêneros de Eriocaulaceae. Acta bot. bras. 4(1): 133-159.

Giulietti, A.M. \& Hensold, N.C. 1991. Synonymization of the genera Comanthera and Carptotepala with Syngonanthus (Eriocaulaceae). Ann. Missouri Bot. Gard. 78: 273-295.

Giulietti, A.M. \& Meikle, R.D. 1982. A problematic transatlantic disjunction in Paepalanthus (Eriocaulaceae). Kew Bull. 37(2): 291-293.

Giulietti, N.; Giulietti, A.M.; Pirani, J.R \& Menezes, N.L. 1988. Estudos em sempre-vivas: importância econômica do extrativismo em Minas Gerais, Brasil. Act. bot. bras. 1(2): 179-194.

Hare, C.L. 1950. The structure and development of Eriocaulon septangulare With. J. Linn. Soc., Bot. 53: $422-448$

Hensold, N.C. 1988. Morphology and systematics of Paepalanthus subg. Xeractis (Eriocaulaceae). Syst. Bot. Monogr. 23.

Hensold, N.C. \& Giulietti, A.M. 1991. Revision and redefinition of the genus Rondonanthus Herzog (Eriocaulaceae). Ann. Missouri Bot. Gard. 78: 441-459.

Lieth, H. 1974. Phenology and seasonality modeling. Ecological Studies 8. Springer Verlag. Berlin.

Mantovani, W. \& Martins, F.R. 1988. Variaçōes fenológicas das espécies do cerrado da Reserva Biológica de Mogi Guaçu, Estado de São Paulo. Rev: Bras. Bot. 11:101-112.

Moldenke, H.N. \& Smith, L.B. 1976. Eriocauláceas. in R. Reitz, (ed.) Flora Ilustrada Catarinense, Herbário "Barbosa Rodrigues", Itajaí. 
Pio Corrêa, M. 1984. Dicionário das plantas úteis do Brasil e das exóticas cultivadas v. 6. Instituto Brasileiro de Desenvolvimento Florestal. Rio de Janeiro.

Ruhland, W. 1903. Eriocaulaceae in A. Engler (ed.), Das Pflanzenr. 4(3): 1-294. Wilhelm Engelmann. Leipzig.

Sano, P.T. 1993. O gênero Paepalanthus Kunth sect. Actinocephalus Koern. (Eriocaulaceae) na Serra do Cipó, Minas Gerais, Brasil: Taxonomia e Fenologia. Dissertação de Mestrado. São Paulo: Universidade de São Paulo.

Souza, V.C. 1990. Scrophulariaceae da Serra do Cipó, Minas Gerais, Brasil. Dissertação de Mestrado. São Paulo: Universidade de São Paulo.

Tenório, E.C. 1969. Fenologia de gramíneas. Anais XX Congresso Nacional de Botânica, Goiânia: 231 241. Goiânia.

Vilegas, W.; Roque, N.F.; Salatino, A.; Giesbrecht, A.M. \& Davino, S. 1990. Isocoumarin from Paepalanthus bromelioides. Phytochemistry 29: 2299-2301.

Weberling, F. 1989. Morphology of flowers and inflorescences. Cambridge University Press. Cambridge. 